\title{
Reducing Protein Corona Formation and Enhancing Colloidal Stability of Gold Nanoparticles by Capping with Silica Monolayers
}

\author{
Jesús Mosquera, ${ }^{* \dagger}$ Isabel García, ${ }^{\dagger}$ Malou Henriksen-Lacey,${ }^{\dagger}$ Guillermo González-Rubio ${ }^{\dagger}$, and Luis M. \\ Liz-Marzán*ił \\ †CIC biomaGUNE and CIBER-BBN, Paseo de Miramón 182, 20014 Donostia-San Sebastián, Spain
}

Ikerbasque, Basque Foundation for Science, 48013 Bilbao, Spain

\begin{abstract}
The design of highly stable gold nanoparticles with low affinity for biomolecules present in physiological media is a central challenge in nanomedicine. Herein we describe a simple coating approach to stabilize gold nanoparticles, based on the formation of a monolayer of silica around nanoparticles pre-stabilized with a small amount of poly(ethylene glycol) ligands. Once the monolayer has been formed, gold nanoparticles are highly stable against adsorption of competing thiol ligands, cyanide digestion and even lyophilization, and their surface can be readily functionalized using silane coupling agents. When the coated gold nanoparticles are incubated with fetal bovine serum, protein adsorption is significantly reduced, as compared to similar gold nanoparticles fully covered with PEG, which is the most common strategy to avoid protein corona formation.
\end{abstract}

Gold nanoparticles (Au NPs) constitute one of the most remarkable products derived from nanotechnology research. ${ }^{1}$ Mainly due to the lack of toxicity of metallic gold, easy functionalization and plasmonic properties, Au NPs have found a variety of biomedical applications such as sensing, image enhancement, and drug delivery.,3 Unfortunately, their application in medicine is still hampered owing to limited stability in biological environments. When Au NPs are immersed in biological media, their physicochemical properties dramatically change with time due to degradation or removal of the original coating agents, leading to undesired effects. ${ }^{4} \mathrm{An}$ additional challenge is the formation of a so-called "protein corona" (PC), i.e. the NP surface gets crowded with biomolecules, mainly proteins. In general, PC formation dramatically modifies the original physicochemical properties of the NPs, blocking targeting capabilities and reducing cellular uptake. 5,6 The most common approach towards reducing PC formation on NPs comprises covering their surface with poly(ethylene glycol) (PEG) chains. ${ }^{7}$ However, the majority of reports so far show that $\mathrm{PC}$ formation is only partly inhibited by PEG coating. ${ }^{8}$ An interesting alternative to PEG for reducing PC formation is based on the use of zwitterionic ligands, which have been shown to completely inhibit PC formation on small Au NPs. ${ }^{9}$

Common methods to enhance the biological stability of $\mathrm{Au}$ NPs include capping with thiolated organic polymers and coating with silica shells. ${ }^{10,11}$ The former method takes advantage of the high affinity between gold and thiols, to attach polymers that enhance colloidal stability in aqueous media, such as thiolated PEG. The main limitation of this strategy is that organic ligands can be removed from the surface of the NP due to exchange with natural thiolated molecules present in biological environments, or to oxidative desorption and enzymatic degradation mechanisms. ${ }^{12,13}$ As an alternative, silica coating is ideal to protect the gold core from the direct attachment of biomolecules, but in general silica shells are unstable in biological media and their degradation leads to exposure of the gold surface. ${ }^{14,15}$ In addition, silica shells smaller than $10 \mathrm{~nm}$ in thickness are usually too porous, thus unable to efficiently shield the gold surface from external agents. Therefore, thick silica shells are typically used, which increase the overall size of the Au NPs, in some cases leading to particle sizes that are too large for certain biological applications. ${ }^{16,17}$

3-mercaptopropyltrimethoxysilane (MPTMS) is a commercially available molecule, which is often employed to functionalize silica surfaces with thiol groups, ${ }^{18}$ or as a primer for the deposition of silica shells on metal nanoparticles. ${ }^{19}$ Interestingly, it has also been described that, when a metallic surface containing a high density of MPTMS is treated with an aqueous basic solution, the silane groups can cross-link through the formation of siloxane bonds, thereby forming a polymeric silica monolayer over the metallic surface. ${ }^{20,21}$ On these grounds, we envisioned the implementation of a similar process toward stabilizing $\mathrm{Au}$ NPs via the formation of a silica monolayer, which would be connected to the NP surface by a large number of thiol-metal bonds, resulting in Au NPs with particularly high colloidal and chemical stability. 22,23

We herein demonstrate the validity of this approach with one of the most extensively used Au NPs, namely $15 \mathrm{~nm}$ diameter (Figure S1, SI), citrate-stabilized Au nanospheres (Au NSs), synthesized by the Turkevich method ${ }^{24}$ (NS-1). Contrary to the case when MPTMS is used for silica deposition, a high ligand density of MPTMS over the Au NP surface is needed to achieve silica monolayer formation. In a first attempt to have a high density ligand coverage around NS-1, an excess of MPTMS (calculated to provide 200 molecules per $\mathrm{nm}^{2}$ ) was directly added to a Au NP hydrosol, but this resulted in immediate aggregation. This lack of colloidal stability most likely stems from the fact that MPTMS is too hydrophobic and too small to stabilize medium-sized Au NPs in water.

Our group recently reported that pre-stabilization of metal NPs with a small amount of PEG-SH $(\mathrm{MW}=5000)$ enables NP surface capping with small hydrophobic molecules, with no loss of colloidal stability. ${ }^{25}$ Therefore, 0.8 molecules of PEG-SH per $\mathrm{nm}^{2}$ of Au surface were added to NS-1, and the suspension was stirred for 10 min (Figure 1, NS-2). After this pre-coating step, a large excess of MPTMS (600 molecules per $\mathrm{nm}^{2}$ ) was added in chloroform ( $1 \mathrm{~mL}$ per $\mathrm{mL}$ of water) and the biphasic system was vigorously stirred until completing the transfer of NPs from water into the organic solvent (NS-3). The water layer was then removed and the particles were washed by centrifugation to remove excess MPTMS. The resulting trimethoxysilane moiety 
covering the surface of NS-3 was subsequently hydrolyzed by addition of $5 \mathrm{mM} \mathrm{NaOH}$ in a $2: 1$ methanol/water mixture, yielding a polymeric monolayer. Finally, the mixture was centrifuged and the NPs dispersed in pure water, yielding Au NPs covered by a hybrid polymeric coating (NS-4). Importantly, no significant changes were observed in the intensity and position of the localized surface plasmon resonance (LSPR) band (at $520 \mathrm{~nm}$ ) after several weeks, which indicates the long-term colloidal stability of the as-prepared $\mathrm{Au}$ NPs. We hypothesize that the presence of a few PEG chains on the NP surface provides additional colloidal stability by a combination of the electrostatic stability imposed by the silica surface, with the steric shielding by PEG chains in NS-4.

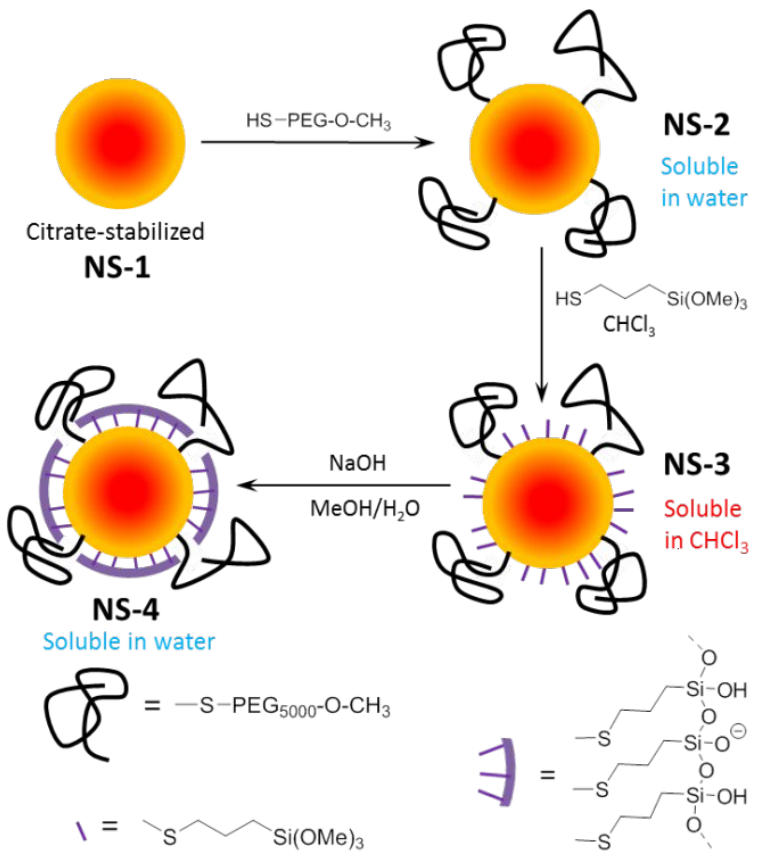

Figure 1. Schematic representation of the preparation of $\mathrm{PEG} / \mathrm{SiO}_{2}$ - shielded Au NPs (NS-4).

Sample NS-4 was characterized by transmission electron microscopy (TEM), UV-vis spectroscopy and zeta potential (Zp) measurements (Figures S2 and S3, SI). TEM images in combination with absorbance spectra confirmed that NS-4 particles remained colloidally stable during synthesis. As expected, the silica monolayers could not be observed by TEM due to their small size, whereas the negative value of $\mathrm{Zp}$ in aqueous solution $(-26 \pm 1 \mathrm{mV})$ proved the presence of deprotonated silanol groups on the NP surface. Furthermore, ${ }^{1} \mathrm{H}-\mathrm{NMR}$ after NS-3 etching with potassium cyanide - note that NS-3 is less stable against cyanide digestion than NS-4 allowed us to determine a MPTMS:PEG molar ratio on the NS3 NP surface of ca. 70:1 (Figure S4, SI). A similar Au NP colloid was synthesized, but its surface was saturated with only PEG-SH (MW=5,000), which is the most commonly used ligand for $\mathrm{Au}$ NPs in biological applications, due to high colloidal stability and biocompatibility (NS-PEG). ${ }^{26}$ These NPs were used as a reference to test the stability and biological behavior of NS-4 (vide infra).

Having at hand both NS-PEG and NS-4, we compared their chemical stability using three different assays: cyanide digestion, dithiothreitol (DTT) competition, and lyophilization. The absorbance at $400 \mathrm{~nm}\left(\mathrm{Abs}_{400}\right)$, which is related to the content of gold in the suspension, ${ }^{27}$ as well as the aggregation factor defined as the ratio between the optical densities at 740 $\mathrm{nm}$ and $520 \mathrm{~nm}\left(\mathrm{AF}=\mathrm{Abs}_{740} / \mathrm{Abs}_{520}\right)$, were used to calculate the chemical and colloidal stability of the NPs, respectively. Cyanide digestion is a general way to evaluate the access of the gold surface to chemical attacks. ${ }^{28}$ When NS-PEG was incubated at room temperature with $10 \mathrm{mM} \mathrm{KCN}$, ca. $85 \%$ of the gold NPs were found to oxidize within one hour, based on the value of $\mathrm{Abs}_{400}$ (Figure 2, left). Conversely, when the experiment was repeated with NS-4, the UV-vis spectrum remained unchanged after $1 \mathrm{~h}$ of incubation, and only a $13 \%$ decrease was observed after $24 \mathrm{~h}$ (Figure 2, right). Remarkably, it was necessary to heat NS-4 at $90{ }^{\circ} \mathrm{C}$ for $2 \mathrm{~h}$, to achieve complete damping of the LSPR, which demonstrates the high stability of these Au NPs. Next we assessed the ability of DTT, a small organic molecule with two thiol groups, to displace ligands from the $\mathrm{Au} \mathrm{NP}$ surface and cause irreversible aggregation. ${ }^{29}$ We thus incubated both NS-PEG and NS-4 with $5 \mathrm{mM}$ DTT for $24 \mathrm{~h}$, at room temperature. Although both NPs remained relatively stable in this assay, NS-4 $(\mathrm{AF}=0.09)$ proved again to display a higher stability than NS-PEG $(\mathrm{AF}=0.21)$ (Figure S12, SI).
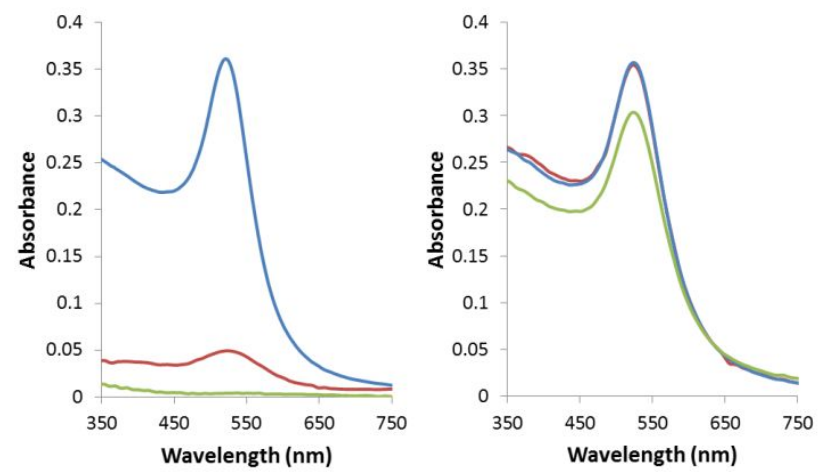

Figure 2. UV-vis spectra of NS-PEG (left) and NS-4 (right) upon cyanide digestion (10 $\mathrm{mM}$ in water), at $0 \mathrm{~h}$ (blue), $1 \mathrm{~h}$ (red), and 24 $\mathrm{h}$ (green). The initial concentration of gold was $0.1 \mathrm{mM}$ in both cases.

Lyophilization has proven ideal for long-term storage of biomolecules and nanoformulations. Unfortunately, both freezing and sublimation processes during lyophilization can induce stress to the $\mathrm{Au}$ NPs and result in undesired aggregation. ${ }^{30}$ In fact, there are only few examples of "mediumsized" Au NPs that remain stable following lyophilization. ${ }^{31-33}$ Encouraged by the observed high stability of NS-4 in the previous assays, we compared the stability of NS-4 and NSPEG upon lyophilization ([Au $]=0.1 \mathrm{mM}, 1 \mathrm{~mL}, 24 \mathrm{~h})$. The results show that, NS-4 was readily redispersed in water after the process, with a decrease in $\mathrm{Abs}_{400}$ as small as 4\%, whereas a reduction of 53\% was found in the signal for NS-PEG (Figure 3). Furthermore, while in NS-4 the AF increased by less than 1.2 times, the AF for NS-PEG after lyophilization was 4 times higher than the initial one. 

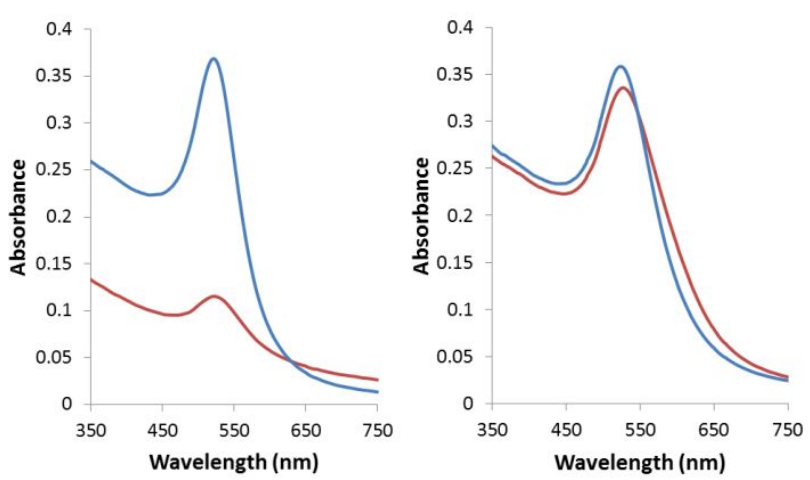

Figure 3. UV-vis absorption spectra of NS-PEG (left) and NS-4 (right), before (blue) and after lyophilization (red). The initial concentration of gold was $0.1 \mathrm{mM}$ in both cases.

Several reports have shown the lack of stability (i.e. dissolution) of silica NPs in biological conditions. This instability is caused mainly by the continous elimination of hydrolysis products that drives backwards the formation equilibrium of polymerized silica, and is particularly augmented at basic $\mathrm{pH} .{ }^{14,15,34}$ Since our strategy is also based on the formation of siloxane bonds, the stability of NS-4 was monitored in the presence of $10 \mathrm{mM} \mathrm{NaOH}(\mathrm{pH}=12)$, which yielded no spectral changes, even after $24 \mathrm{~h}$ of incubation (Figure S13, SI). Equally high colloidal stability was observed when NS-4 was incubated for $24 \mathrm{~h}$ in the presence of salt $(\mathrm{NaCl}$ $100 \mathrm{mM})$, acidic $\mathrm{pH}(\mathrm{pH}=4)$ or culture medium (DMEM supplemented with 10\% FBS) (Figures S13-S15, SI). An additional advantage of silica coating is that silica surfaces can be further functionalized by means of silane coupling agents, which can be easily bound to silanol groups via condensation reactions. ${ }^{35}$ We envisaged that the silanol groups present in NS4 should be able to react with silane coupling agents, just like standard silica. We explored this reactivity using 3aminopropyltriethoxysilane (APTES), which bears an amino group that should switch the negative $\mathrm{Zp}$ value of the original $\mathrm{Au}$ NPs $(-26.5 \pm 0.8 \mathrm{mV})$ into less negative values. As expected, when $1 \mathrm{~mL}$ of NS-4 in EtOH (0.1 mM in gold) was treated with $1 \mathrm{mg}$ of APTES for 5h, followed by three steps of centrifugation and redispersion in water, the $\mathrm{Zp}$ value $(+2 \pm 1$ $\mathrm{mV}$ ) indicated near-neutral particle surfaces (Figure 4).

In addition to gold nanospheres, gold nanorods (Au NRs) are also commonly used for biological applications, since their longitudinal LSPR bands can be readily tailored to be in the near-infrared (NIR) region between 700 and $850 \mathrm{~nm}$, where light penetrates deepest in biological tissues. ${ }^{36} \mathrm{We}$ aimed at testing our strategy with Au NRs, initially stabilized with the cationic surfactant cetyltrimethylammonium bromide (CTAB), and featuring a longitudinal LSPR around $800 \mathrm{~nm}$ (NR-1). Prior to ligand exchange, the CTAB concentration was reduced to 1 $\mathrm{mM}$, so as to facilitate the interaction of thiolated ligands with the gold surface, and then the strategy sketched in Figure 1 was applied. As in the previous case, Au NRs remained colloidally stable during surface modification and the final product (NR-2) was characterized by TEM, UV-vis and $\mathrm{Zp}$ measurements (Figure S8-S9, SI). The chemical stability was again studied by cyanide digestion, DTT competition, and lyophilization. The behavior of NR-2 in all three assays was found to be similar to that of NS-4, with remarkably high stability (Figure S16-S18, $\mathrm{SI})$ ).

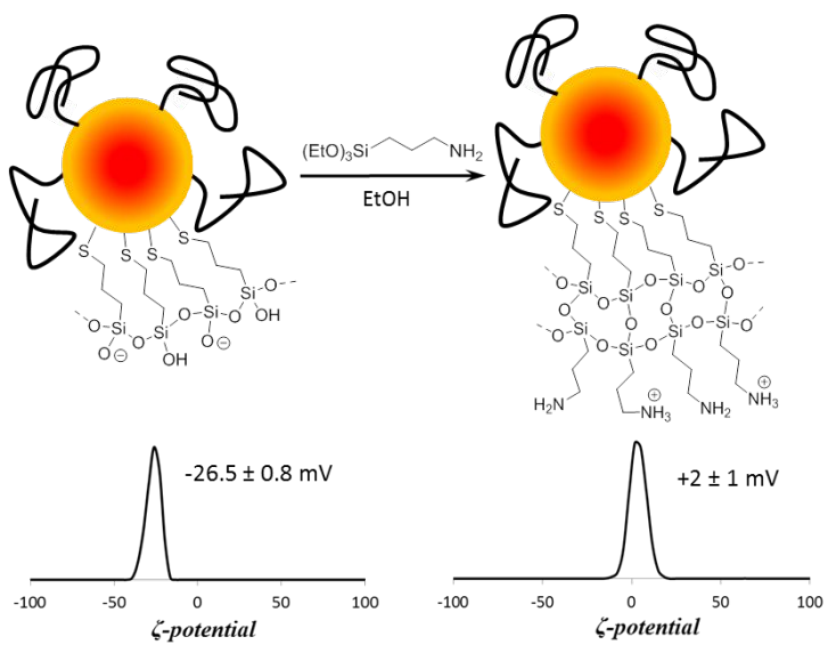

Figure 4. (Top) Schematic representation of the chemical condensation between NS-4 and APTES. (Bottom) Zeta potential measurements of NS-4, before (left) and after (right) the surface condensation reaction.

As mentioned in the introduction above, limited stability and protein corona formation are two major issues that must be considered when using $\mathrm{Au} \mathrm{NPs}$ as biomedical tools. Biomolecules can adsorb onto Au NPs, either through weak interactions yielding a soft corona, or through irreversible interactions forming the so-called hard corona, which largely determines the physiological response. ${ }^{37}$ In order to assess the potential use of this technology for biological applications, we evaluated the formation of hard protein corona over the surface of NS-4, and compared the results to those using NS-PEG, especially considering that PEG ligands are most widely used toward the reduction or inhibition of PC formation. ${ }^{7}$ Thus, we incubated equal concentrations of NPs $\left(1.5 \times 10^{12}\right.$ particles $\left./ \mathrm{mL}\right)$ in diluted FBS $(10 \%$ in PBS) for $1 \mathrm{~h}$, at room temperature. The incubated NPs were separated from non-adsorbed proteins by careful centrifugation $\left(10{ }^{\circ} \mathrm{C}, 19500 \mathrm{~g}\right.$ for $\left.20 \mathrm{~min}\right)$, followed by extensive washing with PBS to remove all unbound proteins, until the supernatant lacked of any detectable protein (see protocol in the SI). Subsequently, adsorbed proteins were isolated from the NPs upon treatment with sodium dodecyl sulfate (SDS) $10 \% / \mathrm{DTT} 500 \mathrm{mM}$, at $90{ }^{\circ} \mathrm{C}$ for $10 \mathrm{~min}$, and subsequently analyzed by SDS polyacrylamide gel electrophoresis (SDS-PAGE) (Figure 5).

The former experiments revealed that four-fold larger amount of proteins were bound to NS-PEG (Figure 5, lane 1) than to NS-4 (Figure 5, lane 2). To further confirm this promising result, we synthesized an analog of NR-2, but fully covered with only PEG ligands (NR-PEG), and repeated the same assay. As shown for spheres above, SDS-PAGE results showed that NR-PEG adsorbed three times more hard corona proteins (Figure 5, lane 3) than NR-2 (Figure 5, lane 4). The former protocol was repeated for NS-4 and NS-PEG using 50\% of FBS (instead of $10 \%$ as used in the previous assay). The amount of hard corona for NS-4 was $10 \%$ lower than that for NS-PEG under the same conditions (Figure S19, SI).

We conclude that, a silica monolayer in combination with a few PEG chains is much more efficient than PEG ligands only, towards reducing irreversible protein adsorption. We hypothesize that this result might stem from a synergic effect of the silica monolayer and PEG chains. Even though PEG is a rather hydrophilic polymer, it has been shown that interaction 
of PEG-coated NPs with proteins is largely mediated by hydrophobic interactions. ${ }^{38}$ In our case, the particles only have a small number of PEG chains that are surrounded by a highly hydrophilic silica surface, thereby hindering hydrophobic interactions but still retaining the anti-adhesive properties of PEG. Furthermore, the silica monolayer present on the Au NP also hinders the direct interaction between the gold surface and biomolecules with high affinity for gold, such as those containing thiols or amines.

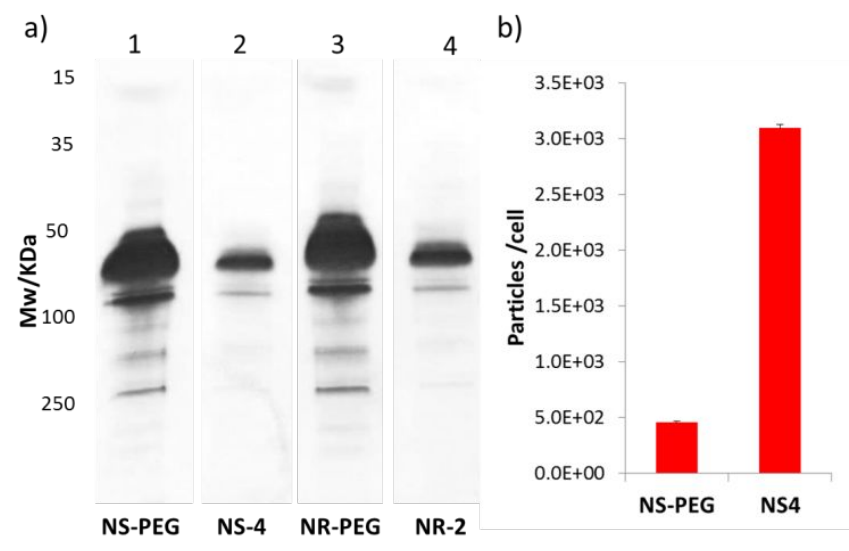

Figure 5. Protein corona and cell internalization studies. a) Comparison of proteins immobilized onto the surface of nanoparticles exposed to $10 \%$ FBS in PBS. The amount of protein was quantified using ImageJ and normalized against the result for NS-PEG. Lane 1: proteins eluted from NS-PEG (100\%). Lane 2: proteins eluted from NS-4 (23\%). Lane 3: proteins eluted from NRPEG (97\%). Lane 4: proteins eluted from NR-2 (31\%). The molecular weights of the proteins in the standard marker are shown on the right for reference. b) Cellular uptake by HeLa cells of NSPEG and NS4 incubated in the presence of DMEM medium with $10 \%$ FBS for $24 \mathrm{~h}$. After two washing steps with PBS the quantity of gold per cell was determined by ICP-MS.

One of the most important consequences of PC formation on NPs is the reduction of their cellular uptake. ${ }^{39} \mathrm{We}$ carried out preliminary cell internalization studies, using HeLa cells to test whether the partial PC inhibition of our approach affects the cell internalization properties of $\mathrm{Au}$ NPs. NS-4 and NS-PEG ( $7 \times 10^{11}$ particles $\left./ \mathrm{mL}\right)$ were incubated with HeLa cells for $24 \mathrm{~h}$ in DMEM medium containing $10 \%$ FBS, followed by quantification of the cellular uptake by inductive coupled plasma-mass spectrometry (ICP-MS). Whereas the incubation of NS-PEG led to the internalization of only $455 \pm 10$ particles/cell, in the case of NS4 the cellular uptake increased by up to 7 -fold ( $3093 \pm 30$ particles/cell, Figure 5b). Thus, this result corroborates the importance of the $\mathrm{PC}$ in the biological behavior of NPs, and how the reduction of the PC can be used to dramatically increase the cellular uptake of Au NPs.

In conclusion, we have developed an efficient coating approach to stabilize $\mathrm{Au}$ NPs, based on the thiolated ligand MPTMS. Once MPTMS is bound to the surface of Au NPs, the silane moieties can be hydrolyzed under basic conditions, forming a polymeric silica monolayer that renders extremely high NP stability. This strategy results in NPs combining the main advantages of organic polymers and traditional silica coatings. Similar to organic polymers, this silica monolayer coating is stable in aqueous media, even at very basic $\mathrm{pH}$, and does not lead to a significant size increase. In addition, the silica coating makes $\mathrm{Au}$ NPs impervious against cyanide digestion, ligand exchange, and lyophilization, and their outer surface can be readily further functionalized using silane coupling agents. Furthermore, our studies relating to protein corona formation showed a significant enhancement of the protecting efficacy of $\mathrm{PEG} / \mathrm{SiO}_{2}$ coating toward reduction of $\mathrm{PC}$ formation, which results in significant enhancement of cellular uptake, when compared to traditional PEG-coated NPs. Therefore, these results open the way towards the design of $\mathrm{Au}$ NPs whose physicochemical properties remain unaltered by the presence of biological molecules, even after long exposure times.

\section{ASSOCIATED CONTENT}

The Supporting Information is available free of charge on the ACS Publications website.

Supplemental Figures S1-S19, synthesis and characterization, experimental procedures and cell studies.

\section{AUTHOR INFORMATION}

\author{
Corresponding Author \\ * e-mail: jmosquera@cicbiomagune.es (JM); \\ llizmarzan@cicbiomagune.es (LML-M).
}

\section{ACKNOWLEDGMENT}

This work has been funded by the Spanish MINECO (Grant MAT2017-86659-R). J.M. acknowledges receipt of a Juan de la Cierva-fellowship (FJCI-2015-25080).

\section{REFERENCES}

(1) Yeh, Y.-C.; Creran, B.; Rotello, V. M. Gold Nanoparticles: Preparation, Properties, and Applications in Bionanotechnology. Nanoscale 2012, 4, 1871-1880.

(2) Giljohann, D. A.; Seferos, D. S.; Daniel, W. L.; Massich, M. D.; Patel, P. C.; Mirkin, C. A. Gold Nanoparticles for Biology and Medicine. Angew. Chem. Int. Ed. Eng. 2010, 49, 3280-3294.

(3) García, I.; Mosquera, J.; Plou, J. ; Liz-Marzán, L. M. Plasmonic Detection of Carbohydrate-Mediated Biological Events. Adv. Opt. Mater. 2018, 6, 1800680.

(4) Feliu, N.; Docter, D.; Heine, M.; Pino, P. del; Ashraf, S.; Kolosnjaj-Tabi, J.; Macchiarini, P.; Nielsen, P.; Alloyeau, D.; Gazeau, F.; et al. In Vivo Degeneration and the Fate of Inorganic Nanoparticles. Chem. Soc. Rev. 2016, 45, $2440-2457$.

(5) Charbgoo, F.; Nejabat, M.; Abnous, K.; Soltani, F.; Taghdisi, S. M.; Alibolandi, M.; Thomas Shier, W.; Steele, T. W. J.; Ramezani, M. Gold Nanoparticle Should Understand Protein Corona for Being a Clinical Nanomaterial. J. Contr. Release 2018, 272, 39-53.

(6) Mosquera, J.; García, I.; Liz-Marzán, L. M. Cellular Uptake of Nanoparticles versus Small Molecules: A Matter of Size. ACC. Chem. Res. 2018, 51, 2305-2313.

(7) Gref, R.; Lück, M.; Quellec, P.; Marchand, M.; Dellacherie, E.; Harnisch, S.; Blunk, T.; Müller, R. 'Stealth' Corona-Core 
Nanoparticles Surface Modified by Polyethylene Glycol (PEG): Influences of the Corona (PEG Chain Length and Surface Density) and of the Core Composition on Phagocytic Uptake and Plasma Protein Adsorption. Colloid Surf. B Biointerfaces 2000, 18, 301-313.

(8) Aggarwal, P.; Hall, J. B.; McLeland, C. B.; Dobrovolskaia, M. A.; MCNeil, S. E. Nanoparticle Interaction with Plasma Proteins as It Relates to Particle Biodistribution, Biocompatibility and Therapeutic Efficacy. Adv. Drug Deliv. Rev. 2009, 61, 428-437.

(9) Moyano, D. F.; Saha, K.; Prakash, G.; Yan, B.; Kong, H.; Yazdani, M.; Rotello, V. M. Fabrication of Corona-Free Nanoparticles with Tunable Hydrophobicity. ACS Nano 2014, 8, 6748-6755.

(10) Manson, J.; Kumar, D.; Meenan, B. J.; Dixon, D. Polyethylene Glycol Functionalized Gold Nanoparticles: The Influence of Capping Density on Stability in Various Media. Gold Bull. 2011, 44, 99105.

(11) Hanske, C.; Sanz-Ortiz, M. N.; Liz-Marzán, L. M. Silica-Coated Plasmonic Metal Nanoparticles in Action. Adv. Mater. 2018, 30, 1707003 .

(12) Schoenfisch, M. H.; Pemberton, J. E. Air Stability of Alkanethiol Self-Assembled Monolayers on Silver and Gold Surfaces. J. Am. Chem. Soc. 1998, 120, 4502-4513.

(13) Kreyling, W. G.; Abdelmonem, A. M.; Ali, Z.; Alves, F.; Geiser, M.; Haberl, N.; Hartmann, R.; Hirn, S.; de Aberasturi, D. J.; Kantner, K.; et al. In Vivo Integrity of Polymer-Coated Gold Nanoparticles. Nat. Nanotechnol. 2015, 10, 619-623.

(14) Croissant, J. G.; Fatieiev, Y.; Khashab, N. M. Degradability and Clearance of Silicon, Organosilica, Silsesquioxane, Silica Mixed Oxide, and Mesoporous Silica Nanoparticles. Adv. Mater. 2017, 29, 1604634 .

(15) Shi, Y.; Hélary, C.; Haye, B.; Coradin, T. Extracellular versus Intracellular Degradation of Nanostructured Silica Particles. Langmuir 2018, 34, 406-415.

(16) Guerrero-Martínez, A.; Pérez-Juste, J.; Liz-Marzán, L. M. Recent Progress on Silica Coating of Nanoparticles and Related Nanomaterials. Adv. Mater. 2010, 22, 11821195.

(17) Liu, S.; Han, M.-Y. Silica-Coated Metal Nanoparticles. Chem. - Asian J. 2010, 5, $36-45$.

(18) Ayala-Orozco, C.; Liu, J. G.; Knight, M. W.; Wang, Y.; Day, J. K.; Nordlander, P.; Halas, N. J. Fluorescence Enhancement of Molecules Inside a Gold Nanomatryoshka. Nano Lett. 2014, 14, 2926-2933.

(19) Shah, K. W.; Sreethawong, T.; Liu, S.-H.; Zhang, S.-Y.; Tan, L. S.; Han, M.-Y. Aqueous Route to Facile, Efficient and Functional Silica Coating of Metal Nanoparticles at Room Temperature. Nanoscale 2014, 6, 11273-11281.

(20) Vakarelski, I. U.; McNamee, C. E.; Higashitani, K. Deposition of Silica Nanoparticles on a Gold Surface via a Self-Assembled Monolayer of (3-
Mercaptopropyl) Trimethoxysilane. Colloid Surf. A 2007, 295, 16-20.

(21) Thompson, W. R.; Cai, M.; Ho, M.; Pemberton, J. E. Hydrolysis and Condensation of Self-Assembled Monolayers of (3-Mercaptopropyl) Trimethoxysilane on Ag and Au Surfaces. Langmuir 1997, 13, 2291-2302.

(22) Wang, W.; Ji, X.; Du, L.; Mattoussi, H. Enhanced Colloidal Stability of Various Gold Nanostructures Using a Multicoordinating Polymer Coating. J. Phys. Chem. C 2017, 121, 22901-22913.

(23) Chen, Y.; Cho, J.; Young, A.; Taton, T. A. Enhanced Stability and Bioconjugation of Photo-Cross-Linked Polystyrene-Shell, Au-Core Nanoparticles. Langmuir 2007, 23, $7491-7497$.

(24) Kimling, J.; Maier, M.; Okenve, B.; Kotaidis, V.; Ballot, H.; Plech, A. Turkevich Method for Gold Nanoparticle Synthesis Revisited. J. Phys. Chem. B 2006, 110, 15700-15707.

(25) Serrano-Montes, A. B.; Jimenez de Aberasturi, D.; Langer, J.; GinerCasares, J. J.; Scarabelli, L.; Herrero, A.; Liz-Marzán, L. M. A General Method for Solvent Exchange of Plasmonic Nanoparticles and Self-Assembly into SERS-Active Monolayers. Langmuir 2015, $31,9205-9213$.

(26) Suk, J. S.; Xu, Q.; Kim, N.; Hanes, J.; Ensign, L. M. PEGylation as a Strategy for Improving Nanoparticle-Based Drug and Gene Delivery. Non-Antigen. Regul. Target. Imaging Ther. 2016, 99, 28-51.

(27) Scarabelli, L.; Grzelczak, M.; LizMarzán, L. M. Tuning Gold Nanorod Synthesis through Prereduction with Salicylic Acid. Chem. Mater. 2013, 25, $4232-4238$.

(28) Hou, W.; Dasog, M.; Scott, R. W. J. Probing the Relative Stability of Thiolate- and Dithiolate-Protected Au Monolayer-Protected Clusters. Langmuir 2009, 25, 12954-12961.

(29) Kang, J. S.; Taton, T. A. Oligothiol Graft-Copolymer Coatings Stabilize Gold Nanoparticles against Harsh Experimental Conditions. Langmuir 2012, 28, 1675116760 .

(30) França, Á.; Pelaz, B.; Moros, M.; Sánchez-Espinel, C.; Hernández, A.; Fernández-López, C.; Grazú, V.; Fuente, J. M. de la; Pastoriza-Santos, I.; Liz-Marzán, L. M.; et al. Sterilization Matters: Consequences of Different Sterilization Techniques on Gold Nanoparticles. Small $2010,6,89-95$.

(31) Feineis, S.; Lutz, J.; Heffele, L.; Endl, E.; Albrecht, K.; Groll, J. ThioetherPolyglycidol as Multivalent and Multifunctional Coating System for Gold Nanoparticles. Adv. Mater. 2018, 30, 1704972 .

(32) Mangeney, C.; Ferrage, F.; Aujard, I.; Marchi-Artzner, V.; Jullien, L.; Ouari, O.; Rékaï, E. D.; Laschewsky, A.; Vikholm, I.; Sadowski, J. W. Synthesis and Properties of Water-Soluble Gold Colloids Covalently Derivatized with Neutral 
Polymer Monolayers. J. Am. Chem. Soc. 2002, 124, 5811-5821.

(33) Gupta, A.; Moyano, D. F.; Parnsubsakul, A.; Papadopoulos, A.; Wang, L.-S.; Landis, R. F.; Das, R.; Rotello, V. M. Ultrastable and Biofunctionalizable Gold Nanoparticles. ACS Appl. Mater. Interfaces 2016, 8, 14096-14101.

(34) Yamada, H.; Urata, C.; Aoyama, Y.; Osada, S.; Yamauchi, Y.; Kuroda, K. Preparation of Colloidal Mesoporous Silica Nanoparticles with Different Diameters and Their Unique Degradation Behavior in Static Aqueous Systems. Chem. Mater. 2012, 24, 1462-1471.

(35) Schmidt, H.; Scholze, H.; Kaiser, A. Principles of Hydrolysis and Condensation Reaction of Alkoxysilanes. J. Non-Cryst. Solids 1984, 63, 1-11.

(36) Stone, J.; Jackson, S.; Wright, D. Biological Applications of Gold Nanorods.
Wiley Interdiscip. Rev. Nanomed. Nanobiotechnol. 2011, 3, 100-109.

(37) Monopoli, M. P.; Åberg, C.; Salvati, A.; Dawson, K. A. Biomolecular Coronas Provide the Biological Identity of Nanosized Materials. Nat. Nanotechnol. 2012, 7, 779786 .

(38) Settanni, G.; Zhou, J.; Suo, T.; Schöttler, S.; Landfester, K.; Schmid, F.; Mailänder, V. Protein Corona Composition of Poly(Ethylene Glycol)- and Poly (Phosphoester)-Coated Nanoparticles Correlates Strongly with the Amino Acid Composition of the Protein Surface. Nanoscale 2017, 9, 2138-2144.

(39) Lesniak, A.; Salvati, A.; SantosMartinez, M. J.; Radomski, M. W.; Dawson, K. A.; Åberg, C. Nanoparticle Adhesion to the Cell Membrane and Its Effect on Nanoparticle Uptake Efficiency. J. Am. Chem. Soc. 2013, 135, 1438-1444. 
Table of Contents Graphic

1
2

3

4

5

6

7

8

9

10

11

12

13

14

15

16

17

18

19

20

21

22

23

24

25

26

27

28

29

30

31

32

33

34

35

36

37

38

39

40

41

42

43

44

45

46

47

48

49

50

51

52

53

54

55

56

57

58

59

60
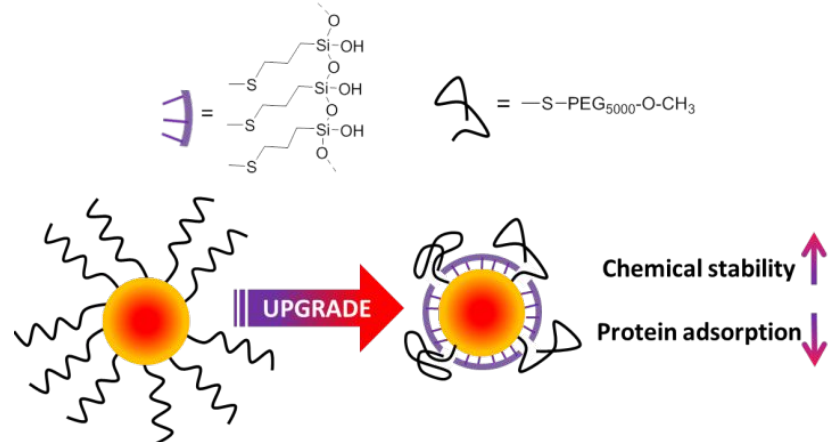